
$\$$ Research Square
Preprints are preliminary reports that have not undergone peer review.
They should not be considered conclusive, used to inform clinical practice,
or referenced by the media as validated information.

\title{
The Inhibition of Long Non-Coding RNA CAR10 and The Regulation of miR-203 Expression Suppresses Cell Viability and Induces Cell Apoptosis in Prostate Cancer
}

\section{Liansheng Zhang}

Soochow University Affiliated Wuxi Ninth Hospital

Yougan Chen

Affiliated Hospital of Jiangnan University

Zhenjie Wang

Xinghua People's Hospital

Qiang Xia ( $\nabla$ xiaqiang062320@163.com )

Soochow University Affiliated Wuxi Ninth Hospital

Research

Keywords: prostate cancer, long non-coding RNA CAR10, microRNA-203.

Posted Date: June 25th, 2020

DOI: https://doi.org/10.21203/rs.3.rs-37761/v1

License: (c) (1) This work is licensed under a Creative Commons Attribution 4.0 International License.

Read Full License 


\section{Abstract}

Background: Prostate cancer (PC) is one of the most common malignant tumors. Recently, it has been reported that long noncoding RNAs (IncRNAs) play key roles in tumor progression. Studies have revealed that long non-coding RNA CAR10 (CAR10) can regulate tumor cell behaviors through sponging miR-203. In this study, we examined the effects of CAR10 in PC cells.

Methods: Firstly, real time-quantitative polymerase chain reaction (qRT-PCR) was used to explore CAR10 expression in tumor tissues, peripheral blood of PC patients, and PC cells. We used the dual-luciferase reporter gene assay to analyze the relationship between CAR10 and miR-203. Moreover, flow cytometry, MTT assay, and western blot assay were used to determine cell apoptosis, cell viability, and apoptosisrelated protein expression.

Results: The results showed that CAR10 expression was remarkably higher in PC samples compared with that of control, and CAR10 regulated miR-203 negatively in PC cells. The qRT-PCR results also showed that miR-203 expression was significantly decreased in PC samples. Moreover, knockdown of CAR10 inhibited PC cell viability and promoted cleaved caspase-3 expression but induced PC cell apoptosis and, reduced pro-caspase-3 expression; miR-203 inhibitor reversed these effects.

Conclusion: Our study found that CAR10 is a potential oncogene in PC and suggests that CAR10 inhibition could inhibit PC cell viability but promote PC cell apoptosis through regulating miR-203 expression. Our results show that CAR10 is a potential target for the treatment of PC.

\section{Introduction}

Prostate cancer (PC) is a common malignant tumor in middle-aged and elderly men. PC is prone to metastasis at an early stage. More than $80 \%$ of patients are at the advanced stage when diagnosed, and they lose the chance of radical surgery. Endocrine therapy is the main treatment for PC patients, but after a median time of 14 to 30 months, almost all patients will develop hormone-resistant PC and enter advanced tumors (1).

Genomic studies have shown that only a small portion of the human genome has the function of encoding proteins, and about $98 \%$ of the entire genome is the long non-coding RNA (IncRNA) which do not participate in encoding proteins (2). IncRNA is a class of RNA molecules and is more than 200 nucleotides in length, it lacks the potential to encode proteins because of lacking the open reading frame (3). With the continuous research on IncRNA, it is found that it is involved in many processes of regulating gene expression, such as chromatin remodeling, gene transcription, post-transcriptional regulation, protein translation, etc (4). And its expression is differential in multiple malignant tumors, which is closely related to biological characteristics such as cell proliferation, migration, invasion, and anti-apoptosis of various tumors and patient prognosis (5). 
LncRNA chromatin-associated RNA 10 (CAR10) was first identified in human fibroblasts during sequencing of chromatin-associated RNAs (CARs), and in cis it can regulate the neighboring genes expression (6). CAR10 is reported to express abnormally in various tumors and play important effects in tumors. For example, CAR10 expression is increased in leiomyomas then the overexpression of CAR10 promote the development of uterine leiomyomas (7). It is also reported that CAR10 can regulate the transcription factor Y-boxbinding protein 1 (YB-1) leading to the cancer cell proliferation (8). Ge et al. reported that CAR10 was upregulated in lung adenocarcinoma tissue, and it could promote lung adenocarcinoma metastasis through down-regulating miR-203 expression (9). Moreover, CAR10 has been confirmed promote cervical cancer development by sponging miR-125b-5p (10). However, till now, the role of CAR10 in PC remains unclear.

MicroRNAs (miRNAs) are 18-24-nucleotide non-coding RNA molecules in the genomes of viruses, protozoa, plants, and animals. miRNA is involved in the regulation of post-transcriptional gene expression, and plays an important role in regulating stem cell proliferation, migration and differentiation, and participates in the regulation of stem cell tissue repair. miRNA mainly specifically binds to the $3^{\prime} U T R$ region of mRNA, and regulates post-transcriptional levels by promoting mRNA degradation or inhibiting mRNA translation (11).

The sponging interaction between IncRNA and miRNA is an important mechanism for IncRNA to exert its anti-tumor or tumor-promoting functions, such as in osteosarcoma, gastric cancer, lung cancer, liver cancer and breast cancer (12-16).

miR-203 is one of the important members of the miRNAs family, and located on human chromosome 14 q32, 33-site (17). Studies have shown that miR-203 is abnormally expressed in a variety of tumor tissues including esophageal carcinoma (down-regulation), osteosarcoma (down-regulation), ovarian cancer (upregulation), and prostate cancer (down-regulation), and is closely related to tumor cell growth, metastasis and invasion (18-23). A recent study reported that miRNA-203 restrains epithelial-mesenchymal transition, invasion and migration of papillary thyroid cancer via downregulating AKT3 expression (24). And it was reported that CAR10 can promote the metastasis of lung adenocarcinoma through down-regulating miR$203(9)$.

The aim of this study was to explore the role of CAR10 in PC cells and its related mechanism.

\section{Materials And Methods}

\section{Clinical samples}

30 patients with prostate cancer and 30 healthy volunteers were included in our study. We get human prostate cancer tissues and its adjacent normal tissues from 30 PC patients (age range: 39-68 years old; T1-T2: 17; T3-T4: 13), and obtained peripheral blood from 30 healthy subjects (all males; age range: $34-$ 67 years old) and 30 PC patients respectively. We collected all the samples and then used liquid nitrogen to store them immediately. Patients with a history or current diagnosis of tumors, hypertension, diabetes, 
urinary tract diseases such, or related infectious or immune diseases were excluded. All patients were informed and offered the consents. Ethics Committee of Soochow University Affiliated Wuxi Ninth Hospital approved our study.

\section{Cell culture}

PC cell lines Du145 (ATCC; ATCC $®$ HTB-81) and PC-3 (ATCC; ATCC $®$ CRL-1435), prostate epithelial cells RWPE-2 (ATCC; ATCC ${ }^{\circledR}$ CRL-11610 ${ }^{\text {TM }}$ ) (from healty human) and HEK-293T cells were purchased from Procell Life Science\&Technology Co., Ltd (Wuhan, Hubei, China). All cells were cultured in Dulbeccos Modified Eagle Medium (DMEM; Invitrogen, Carlsbad, CA, USA) which containing $1 \%$ penicillin/streptomycin and $10 \%$ fetal bovine serum (FBS) at $37{ }^{\circ} \mathrm{C}$ with $5 \% \mathrm{CO}_{2}$.

\section{Real time-quantitative polymerase chain reaction (qRT-PCR) (25)}

Trizol reagent (CWIO, Beijing, China) was used to extract total RNA following the manufacturer's protocol, and Nanodrop ND-2000 spectrophotometer (Thermo Scientific ${ }^{\mathrm{TM}}$, USA) was used to get the RNA quantity. Then we used miDETECT A Track ${ }^{\text {TM }}$ qRT-PCR Kit (Guangzhou RiboBio Co., China) and RevertAid First Strand cDNA Synthesis Kit (Thermo Scientific ${ }^{\mathrm{TM}}$, USA) to reverse-transcribe $2 \mu \mathrm{g}$ total RNA into cDNA to analyze miRNA, IncRNA and mRNA expression respectively. Bio-Rad CFX Connect ${ }^{\text {TM }}$ Real-Time PCR Detection System (Bio-Rad, USA) was used to perform qPCR with 2x SYBR Green qPCR Master Mix (Bimake, China), and U6 and GAPDH were used as the normalization. Primer sequences were listed as following:

U6 forward, 5'-GCTTCGGCAGCACATATACTAAAAT-3';

reverse, 5'-CGCTTCACGAATTTGCGTGTCAT-3';

GAPDH forward, 5'-CTTTGGTATCGTGGAAGGACTC-3';

reverse, 5'-GTAGAGGCAGGGATGATGTTCT-3';

CAR10 forward, 5'-TCAGTGCTGCTCCTGAGAGA-3';

reverse, 5'-CAGCCAGAGACCAGTCATCA-3';

miR-203 forward, 5'-ACACTCCAGCTGGGGTGAAATGTTTA-3';

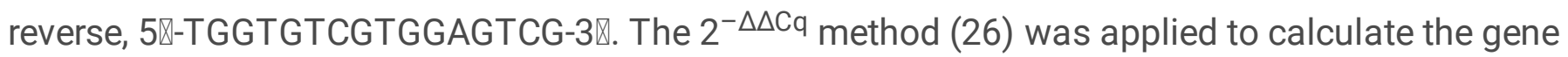
expression.

\section{Dual-luciferase reporter assay (27)}

We inoculated the 293T cells into 24 well plates and cultured overnight, then transfected wildtype/mutant-type CAR10 vector and miR-203 mimic/mimic control respectively into 293T cells using 
Lipofectamine 2000 (Invitrogen) according to the manufacturer's protocol. After $48 \mathrm{~h}$, we followed the instructions and used Dual Luciferase Reporter Assay System (Promega, Madison, WI, USA) to measure the firefly (wavelength: $570 \mathrm{~nm}$ ) and Renilla luciferase (wavelength: $480 \mathrm{~nm}$ ) activities. Renilla luciferase was used as the internal reference.

\section{Cell transfection}

CAR10-siRNA, control-siRNA, miR-203 inhibitor, inhibitor control, CAR10-siRNA+inhibitor control, or CAR10siRNA+ miR-203 inhibitor were obtained from Genechem Co., Ltd, (Shanghai, China), and were transfected into Du145 and PC-3 cells respectively. We used six-well plates to culture cells then used lipofectamine 2000 transfection reagent (Invitrogen, Carlsbad, CA, USA) to perform transfection for $48 \mathrm{~h}$.

\section{Cell viability assay (28)}

We used 96 -well plates to culture cells $\left(5 \times 10^{4}\right.$ cells per well) and then used MTT assay to measure cell viability following the manufacturer's instruction. Briefly, MTT (Beyotime) solution was added into each well and incubated for $4 \mathrm{~h}$ at $37^{\circ} \mathrm{C}$, then the medium was removed and $150 \mu \mathrm{DMSO}$ was added. We used multifunctional micro-plate reader (POLARstar OPTIMA; BMG, Offenburg, Germany) to measure the optical density (OD) at $570 \mathrm{~nm}$.

\section{Flow cytometer analysis (29)}

We collected cells $\left(1 \times 10^{6}\right)$ and washed with PBS and then used $70 \%$ ethanol to fix them at $4^{\circ} \mathrm{C}$ overnight. The percentages of cells that underwent apoptosis were determined by two color analysis. viable cells annexin $\mathrm{V}$ negative and PI negative, early apoptotic cells annexin $\mathrm{V}$ positive and PI negative, necrotic cells annexin $\mathrm{V}$ negative and PI positive, and late apoptotic cells annexin $\mathrm{V}$ positive and PI positive. Then we used Annexin V-FITC (FITC, R\&D Systems Inc., Minneapolis, USA) and propidium iodide (PI, R\&D Systems Inc., USA) to measure the cell apoptosis according to the manufacturer's protocol. At last, the rate of apoptosis was detected using FACSCalibur flow cytometer (BD Technologies) and analyzed using FlowJo 7.6.1 software (FlowJo LLC).

\section{Western blot assay (30)}

Protease inhibitor (Beyotime) and RIPA lysis buffer (Beyotime) were used to extract the protein, and Pierce $^{T M}$ BCA Protein Assay Kit (Thermo Scientific ${ }^{T M}$, USA) was used to quantify the protein concentration. $10 \%$ sodium dodecyl sulfate-polyacrylamide gel electrophoresis was used to separate the equal amounts of protein. Then proteins were transferred to PVDF membranes (Millipore). Then the membranes were incubated for $1 \mathrm{~h}$ with blocking solution containing $5 \%$ nonfat dry milk and then were incubated at $4{ }^{\circ} \mathrm{C}$ overnight with primary antibodies: pro-Caspase3 (Cat no. ab32499; 1:1000; Abcam), cleaved-Caspase3 (Cat no. 9664; 1:1000; CST), GAPDH (Cat no. 5174; 1:1000; CST). After washing for three times using TBST, the membranes were incubated at $37^{\circ} \mathrm{C}$ for $1 \mathrm{~h}$ with horseradish peroxidase-labeled secondary 
antibody (Cat no. 7074; 1:2000; CST). At last, ECL detection reagent (BioRad, USA) and densitometry (BioRad ChemiDoc XRS system) were used to visualize and quantify the signal respectively.

\section{Statistic analysis}

We used GraphPad Prism 6.0 (GraphPad Software, La Jolla, CA, USA) to do the statistics, and used mean \pm standard deviation (SD) to describe the variables. Student's $t$-test or ANOVA analysis followed by Tukeys post-test was performed to get $P$ values, and $P<0.05$ represent statistical differences. We repeated each experiment for three times.

\section{Results}

\section{CAR10 expression was elevated in PC tumor tissues, peripheral blood and cells.}

qRT-PCR was used to explore CAR10 expression in peripheral blood, cancer tissues and the adjacent tissues in PC patients and in the peripheral blood of normal human. We also detected the CAR10 mRNA expression in PC cell lines Du145, PC-3 and normal human prostate epithelial cells RWPE-2. The results showed that CAR10 expressions in PC tissues (Figure 1A; > 4 fold increase), peripheral blood (Figure 1B; > 4 fold increase) and PC cells (Figure 1C; $>4$ fold increase) were increased significantly when compared with the adjacent tissues and normal human prostate epithelial cells RWPE-2.

\section{CAR10 regulated miR-203 negatively in PC cells.}

Figure 2A showed the binding sites between CAR10 and miR-203. In addition, the results of dualluciferase reporter assay revealed that miR-203 mimic had no influence on the luciferase activity of CAR10-MUT-3'UTR, but significantly decreased the luciferase activities of the CAR10-WT-3'UTR clearly (Figure 2B). The data indicated that miR-203 directly targeted CAR10.

\section{miR-203 expression was reduced in PC tumor tissues, peripheral blood and cells.}

qRT-PCR was used to detect miR-203 expression in peripheral blood, cancer tissues and the adjacent tissues in PC patients and in the peripheral blood of normal human. We also detected the miR-203 expression in PC cell lines Du145, PC-3 and normal human prostate epithelial cells RWPE-2. The results showed that the level of miR-203 in PC tissues (Figure 3A; $<-2$ fold decrease), peripheral blood (Figure 3B; $<-2$ fold decrease) and PC cells (Figure $3 \mathrm{C} ;<-2$ fold decrease) were decreased significantly when compared with the adjacent tissues and normal human prostate epithelial cells RWPE-2.

\section{Down-regulation of CAR10 inhibited PC cell viability and induced apoptosis.}

To further confirm the relationship between CAR10 and miR-203 in the regulation of PC cells, CAR10siRNA, control-siRNA, miR-203 inhibitor, inhibitor control, CAR10-siRNA+inhibitor control, or CAR10-siRNA+ miR-203 inhibitor were transfected into Du145 and PC-3 cells, respectively. After transfection for $48 \mathrm{~h}$, we used qRT-PCR to detect the expression of related gene. The results showed that CAR10-siRNA 
significantly reduced CAR10 expression in Du145 and PC-3 cells (Figure 4A and B). miR-203 inhibitor significantly reduced miR-203 expression in Du145 and PC-3 cells (Figure 4C and D). CAR10-siRNA significantly increased miR-203 expression, but co-transfection with miR-203 inhibitor reversed this effect in Du145 and PC-3 cells (Figure 4E and F).

MTT assay and flow cytometry were used to detect PC cell viability and apoptosis, and western blot assay was used to detect the expression of cleaved-caspase 3 and pro-caspase 3 . The results showed that CAR10-siRNA significantly reduced the viability (Figure 5A) but induced apoptosis (Figure 5B and C; apoptosis rate $>50 \%$ ) of Du145 cells, promoted cleaved-caspase 3 protein expression (Figure 5D and E; > 7 fold increase), but reduced pro-caspase3 protein expression (Figure 5D and F) in Du145 cells, while these changes were reversed by co-transfection with miR-203 inhibitor.

Similarly, CAR10-siRNA significantly reduced the viability (Figure 6A), but induced apoptosis (Figure 6B and $\mathrm{C}$ ) of $\mathrm{PC}-3$ cells, promoted cleaved-caspase 3 protein expression (Figure 6D and E), but reduced procaspase3 protein expression (Figure 6D and F) in PC-3 cells, and these changes were reversed by cotransfection with miR-203 inhibitor.

\section{Discussion}

PC is one of the most common malignant tumors in men. It is still easy to metastasize and relapse after hormone therapy and surgery, which seriously affects the quality of life of patients. Therefore, it has positive significance to find reliable molecular markers that reflect the malignancy of PC and suggest clinical prognosis. LncRNAs are one subgroup of non-coding RNAs, it has been proved that IncRNAs have important effects in tumors including PC. For instance, IncRNA PCSEAT can activate EZH2 then promote the viability of PC cells (31). LncRNA SNHG7 can affect miR-503/Cyclin D1 pathway then regulate the viability and cycle progression of PC cells (32). The down-regulation of IncRNA PVT1 can regulate p38 expression then decrease PC cells metastasis (33). In addition, it has been reported that IncRNA HOTAIR and MALAT1 affect transcriptional regulation in PC cells induced by estrogen (34).

It has been reported that CAR10 is a key factor in cancer and other diseases. In this study, we explored the related mechanisms of CAR10 in PC. The results showed that CAR10 expression was increased in PC tissues, peripheral blood and cells significantly when compared with that of control, our results were consistent with the previous reports (7-9). The enhanced expression of CAR10 in both PC tissues and PC peripheral blood implied that CAR10 may be involved in the occurrence and development of prostate cancer.

To further explore the underlying mechanism of how CAR10 regulates PC cells, we used bioinformatic analysis and experimental verification to find that miR-203 was a potential binding miRNA of CAR10, and CAR10 regulated miR-203 directly in PC cells, and knockdown of CAR10 up-regulated miR-203 expression. Thus, we thought that miR-203 expression was correlated with CAR10 negatively in PC. And we then detected miR-203 expression in PC, and the results showed that miR-203 expressions in PC tissues, cells 
and peripheral blood were decreased significantly when compared with that of control. All the results above suggested that CAR10 might promote tumorigenesis of PC through sponging miR-203.

miR-203 has been reported to play a key role in the PC development. For example, miR-203 elevation suppressed PC-3 cell proliferation and ADM resistance but promoted apoptosis through inhibiting MEK1 expression (21). miR-203 plays the role of tumor suppressor gene and regulate RGS17 expression then affect the malignant behavior of PC cells (22). It also has been reported that miR-203/SNAI2 axis had important function in angiogenesis, tumorigenesis, metastasis, stemness in PC (23). Such results indicate that miR-203 is a suppressor in tumors, which is consistent with our results.

Besides, in our study, we next detected the effect of CAR10 expression on the viability and apoptosis of PC cells. The results showed that knockdown of CAR10 could inhibit viability but promote apoptosis, and promoted cleaved-caspase3 expression, but reduced pro-caspase3 expression through regulating miR203 expression in PC cells. Previous study has demonstrated the antiproliferative and apoptosis-inducing effects of miR-203 in prostate cancer (21-23). However, the role of miR-203 in apoptosis and its target genes in PC still need further research. This was a limitation of this study, and we will perform this issue in the future.

\section{Conclusions}

The results of this study showed that CAR10 inhibition could inhibit viability but induce apoptosis in PC cells through up-regulating miR-203 expression. These findings suggested that CAR10 can be a prospective therapeutic target for PC.

\section{Declarations}

\section{Ethics approval and consent to participate}

All patients were informed and offered the consents. Ethics Committee of Soochow University Affiliated Wuxi Ninth Hospital approved our study.

\section{Consent for publication}

All patients agree to publish.

\section{Availability of data and material}

All data sets used and/or generated during the present study are available from the corresponding author on reasonable request.

\section{Competing interests}

The authors declare that they have no competing interests. 


\section{Funding}

No funding was received.

\section{Authors' contributions}

Liansheng Zhang contributed to study design, data collection, statistical analysis, data interpretation and manuscript preparation. Yougan Chen and Zhenjie Wang contributed to data collection and statistical analysis. Qiang Xia contributed to data collection, statistical analysis and manuscript preparation. All authors read and approved the final manuscript.

\section{Acknowledgements}

Not applicable.

\section{References}

1. Moustafa AA, Kim H, Albeltagy RS, El-Habit OH, Abdel-Mageed AB. MicroRNAs in prostate cancer: From function to biomarker discovery. Exp Biol Med (Maywood) 2018; 243: 817-25.

2. Wang Z, Wang J, Wang J, et al. Long noncoding RNAs in gastric cancer: functions and clinical applications. Onco Targets Ther 2016; 9: 681-97.

3. Kopp F, Mendell JT. Functional Classification and Experimental Dissection of Long Noncoding RNAs. Cell 2018; 172: 393-407.

4. Spizzo R, Almeida MI, Colombatti A, Calin GA. Long noncoding RNAs and cancer:a new frontier of translational research. Oncogene 2012; 31: 4577-87.

5. Wapinski O, Chang HY. Non-coding RNAs and human disease. Trends cell Biol 2011; 21: 354-61.

6. Mondal T, Rasmussen M, Pandey G, Isaksson A, Kanduri C. Characterization of the RNA content of chromatin. Genome Res 2010; 20: 899-907.

7. Guo H, Zhang X, Dong R, et al. Integrated analysis of long noncoding RNAs and mRNAs reveals their potential roles in the pathogenesis of uterine leiomyomas. Oncotarget 2014; 5: 8625-36.

8. Wei MM, Zhou YC, Wen ZS, et al. Long non-coding RNA stabilizes the Y-box-binding protein 1 and regulates the epidermal growth factor receptor to promote lung carcinogenesis. Oncotarget 2016; 7: 59556-71.

9. Ge X, Li GY, Jiang L, et al. Long noncoding RNA CAR10 promotes lung adenocarcinoma metastasis via miR-203/30/SNAI axis. Oncogene 2019; 38: 3061-76.

10. Hu T, Zhang Q, Gao L. LncRNA CAR10 Upregulates PDPK1 to Promote Cervical Cancer Development by Sponging miR-125b-5p. Biomed Res Int 2020; 2020: 4351671.

11. Deng B, Wang B, Fang J, et al. MiRNA-203 suppresses cell proliferation, migration and invasion in colorectal cancer via targeting of EIF5A2. Sci Rep 2016; 6: 28301. 
12. Luo W, He H, Xiao W, et al. MALAT1 promotes osteosarcoma development by targeting TGFA viaMIR376A. Oncotarget 2016; 7: 54733-43.

13. Zhang L, Kang W, Lu X, Ma S, Dong L, Zou B. LncRNA CASC11 promoted gastric cancer cell proliferation, migration and invasion in vitro by regulating cell cycle pathway. Cell Cycle 2018; 17: 1886-900.

14. Wang C, Han C, Zhang Y, Liu F. LncRNA PVT1 regulate expression of HIF1a via functioning as ceRNA for miR-199a-5p in non-small cell lung cancer under hypoxia. Molecular Mol Med Rep 2018; 17: 1105-10.

15. Chen Z, Xu D, Zhang T. Inhibition of proliferation and invasion of hepatocellular carcinoma cells by IncRNA-ASLNC02525 silencing and the mechanism. International Journal of Oncology 2017; 51: 851-8.

16. Hu HB, Chen Q, Ding SQ. LncRNA LINC01116 competes with miR-145 for the regulation of ESR1 expression in breast cancer. European Review for Medical \& Pharmacological Sciences 2018; 22 : 1987-93.

17. Tiwari P, Gupta KP. Modulation of miR-203 and its regulators as a function of time during the development of 7,12 dimethylbenz [a] anthracene induced mouse skin tumors in presence or absence of the antitumor agents. Toxicol Appl Pharmacol 2014; 278: 148-58.

18. He R, Wang J, Ye K, Du J, Chen J, Liu W. Reduced miR-203 predicts metastasis and poor survival in esophageal carcinoma. Aging (Albany NY) 2019; 11: 12114-30.

19. Lin W, Zhu X, Yang S, et al. MicroRNA-203 inhibits proliferation and invasion, and promotes apoptosis of osteosarcoma cells by targeting Runt-related transcription factor 2. Biomed Pharmacother 2017; 91: 1075-84.

20. Liu HP, Zhang Y, Liu ZT, Qi H, Zheng XM, Qi LH, Wang JY. MiR-203 regulates proliferation and apoptosis of ovarian cancer cells by targeting SOCS3. Eur Rev Med Pharmacol Sci 2019; 23: 928694.

21. Chen LZ, Ding Z, Zhang Y, He ST, Wang XH. MiR-203 over-expression promotes prostate cancer cell apoptosis and reduces ADM resistance.Eur Rev Med Pharmacol Sci 2018; 22: 3734-41.

22. Zhang LS, Ma HG, Sun FH, Zhao WC, Li G. MiR-203 inhibits the malignant behavior of prostate cancer cells by targeting RGS17. Eur Rev Med Pharmacol Sci 2019; 23: 5667-74.

23. Tian X, Tao F, Zhang B, Dong JT, Zhang Z. The miR-203/SNAI2 axis regulates prostate tumor growth, migration, angiogenesis and stemness potentially by modulating GSK-3 $\beta / \beta-C A T E N I N$ signal pathway. IUBMB Life 2018; 70: 224-36.

24. You A, Fu L, Li Y, Li X, You B. MicroRNA-203 restrains epithelial-mesenchymal transition, invasion and migration of papillary thyroid cancer by downregulating AKT3. Cell Cycle 2020; 19: 1105-21.

25. Chen $L$, Jin $Y$, Wang $L$, et al. Identification of reference genes and miRNAs for qRT-PCR in human esophageal squamous cell carcinoma. Med Oncol 2017; 34: 2.

26. Livak KJ, Schmittgen TD. Analysis of relative gene expression data using real-time quantitative PCR and the 2-DDCt Method. Methods 2001; 25: 402-8. 
27. Clément T, Salone V, Rederstorff M. Dual luciferase gene reporter assays to study miRNA function. Methods Mol Biol 2015; 1296: 187-98.

28. Kumar P, Nagarajan A, Uchil PD. Analysis of Cell Viability by the MTT Assay. Cold Spring Harb Protoc 2018; 2018.

29. Telford WG. Multiparametric Analysis of Apoptosis by Flow Cytometry. Methods Mol Biol 2018; 1678: 167-202.

30. Hnasko TS, Hnasko RM. The Western Blot. Methods Mol Biol 2015; 1318: 87-96.

31. Yang $X$, Wang $L$, Li R, et al. The long non-coding RNA PCSEAT exhibits an oncogenic property in prostate cancer and functions as a competing endogenous RNA that associates with EZH2. Biochem Biophys Res Commun 2018; 502: 262-8.

32. Qi H, Wen B, Wu Q, et al. Long noncoding RNA SNHG7 accelerates prostate cancer proliferation and cycle progression through cyclin D1 by sponging miR-503. Biomed Pharmacother 2018; 102: 326-32.

33. Wan B, Wu HY, Lv DJ, et al. Downregulation of IncRNA PVT1 expression inhibits proliferation and migration by regulating p38 expression in prostate cancer. Oncol Lett 2018; 16: 5160-6.

34. Aiello A, Bacci L, Re A, et al. MALAT1 and HO-TAIR long non-coding RNAs play opposite role in estrogen-mediated transcriptional regulation in prostate cancer cells. Sci Rep 2016; 6: 38414.

\section{Figures}
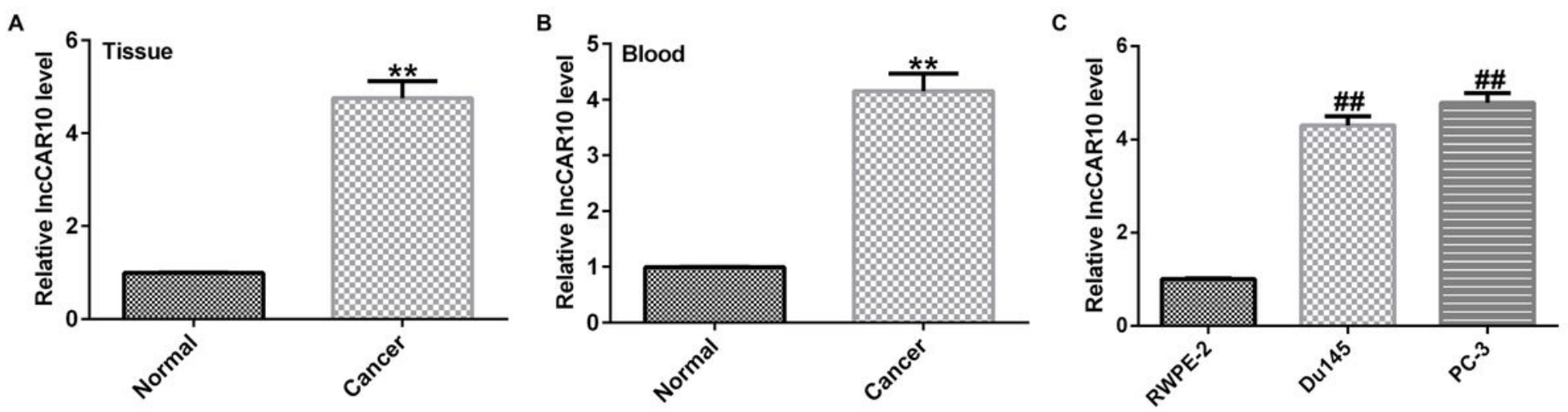

\section{Figure 1}

The expression of CAR10 in PC tumor tissues, peripheral blood and cells. We used qRT-PCR to detect the expression of CAR10 in cancer tissues, peripheral blood and the adjacent tissues in PC patients and in peripheral blood of normal human. We also detected the CAR10 mRNA expression in PC cell line Du145, PC-3 cells and normal human prostate epithelial cells RWPE-2. A: the expression of CAR10 in PC tissues. B: the expression of CAR10 in peripheral blood of PC patients. C: the expression of CAR10 in PC cells. Data were reported as mean $\pm S D$. ${ }^{*} p<0.01$ vs. Normal group; \#\#p<0.01 vs. RWPE-2. 
A

\section{hsa-miR-203 5' $\quad$... GUGAGAUGUACUACUCAUUUCAA... CAR10 3' GAUCACCAGGAUUUGUAAAGUG}

B

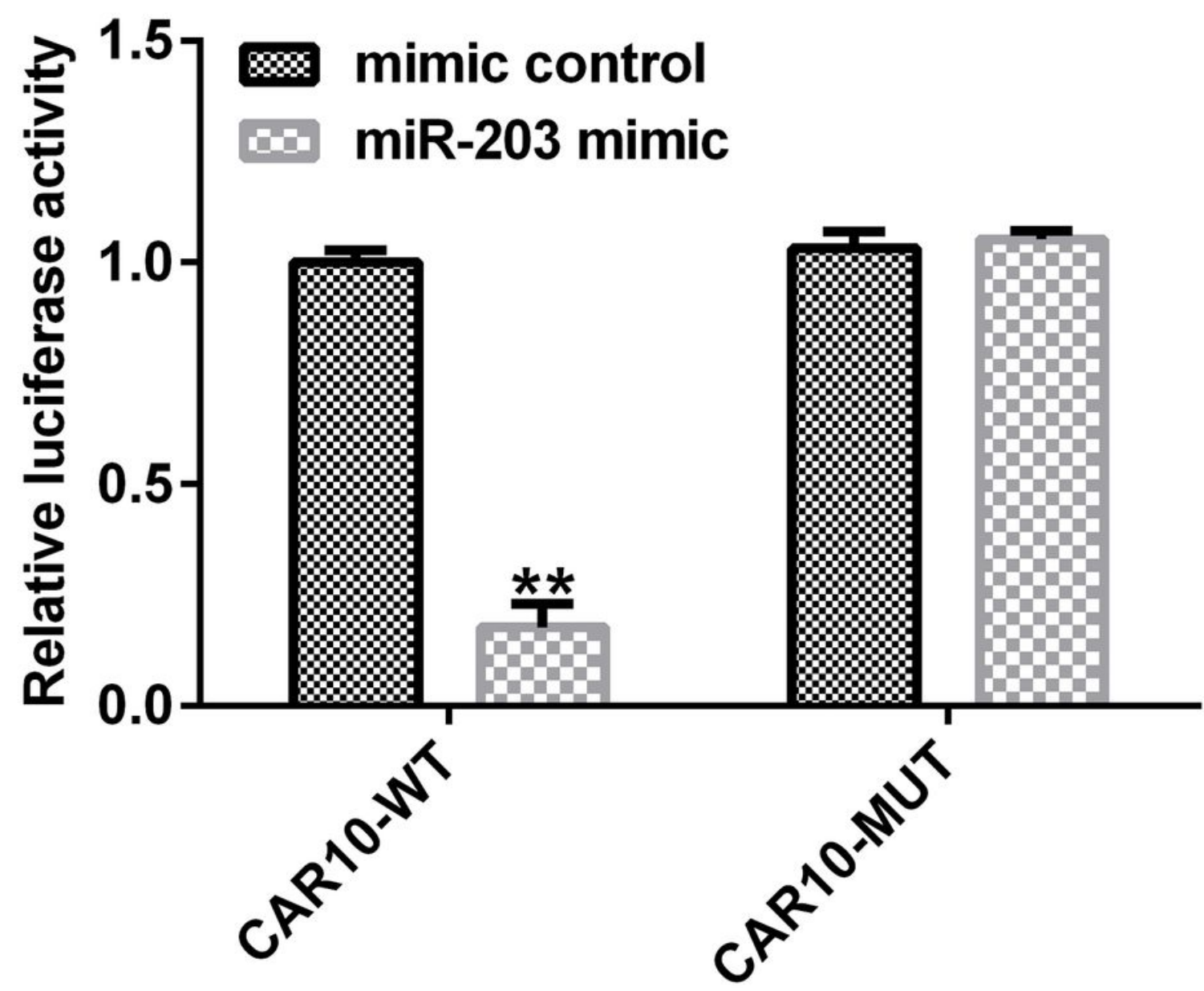

Figure 2

miR-203 directly targeted CAR10. A: the potential binding site between CAR10 and miR-203. B: the Dualluciferase reporter assay verified that miR-203 was a direct target of CAR10. Data were reported as mean $\pm S D$. ${ }^{\star \star} p<0.01$ vs. mimic control group. 
A

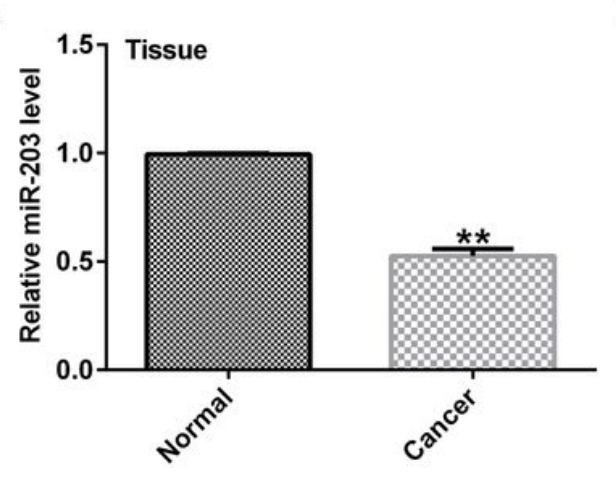

B

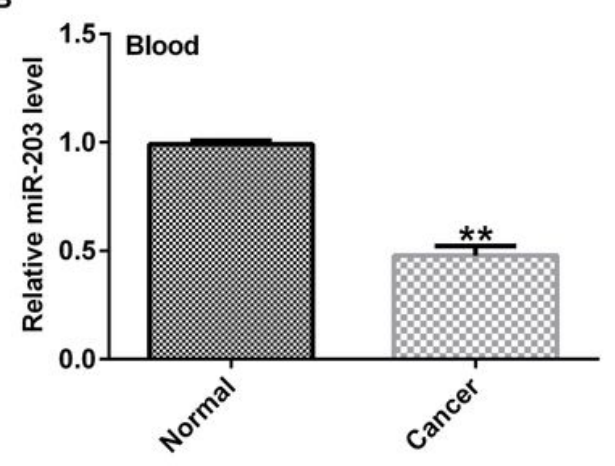

C

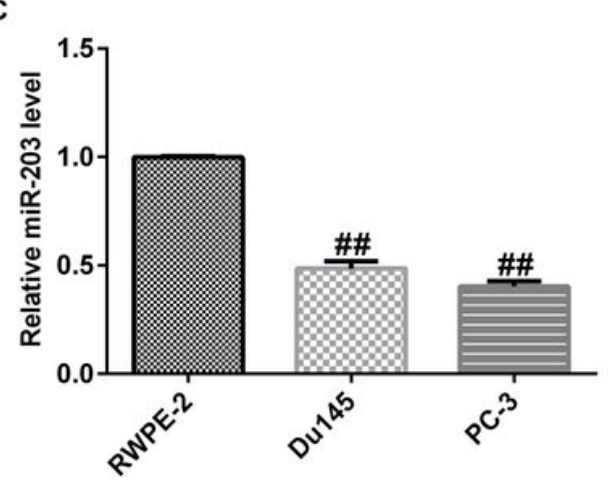

Figure 3

The expression of miR-203 in PC tumor tissues, peripheral blood and cells. We used qRT-PCR to detect the expression of miR-203 in cancer tissues, peripheral blood and the adjacent tissues in PC patients and in peripheral blood of normal human. We also detected the miR-203 mRNA expression in PC cell line Du145, PC-3 cells and normal human prostate epithelial cells RWPE-2. A: the expression of miR-203 in PC tissues. B: the expression of miR-203 in peripheral blood of PC patients. C: the expression of miR-203 in PC cells. Data were reported as mean $\pm S D$. ${ }^{*} p<0.01$ vs. Normal group; \#\#p<0.01 vs. RWPE-2. 
A

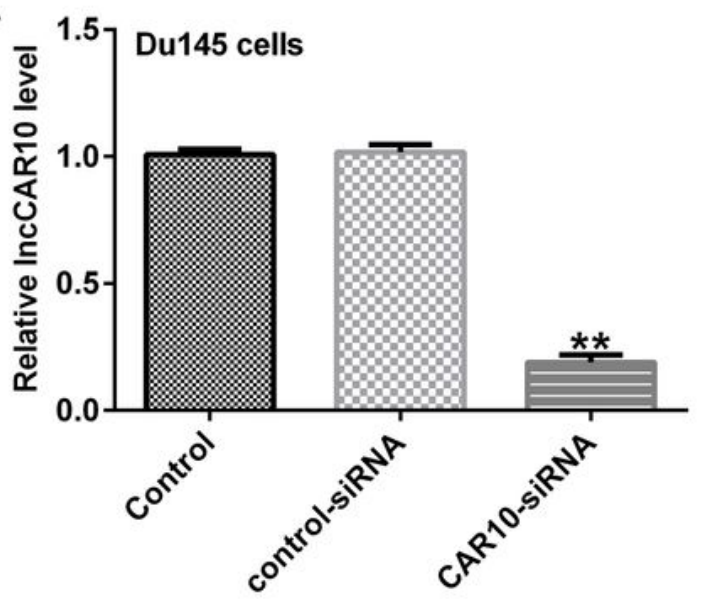

C

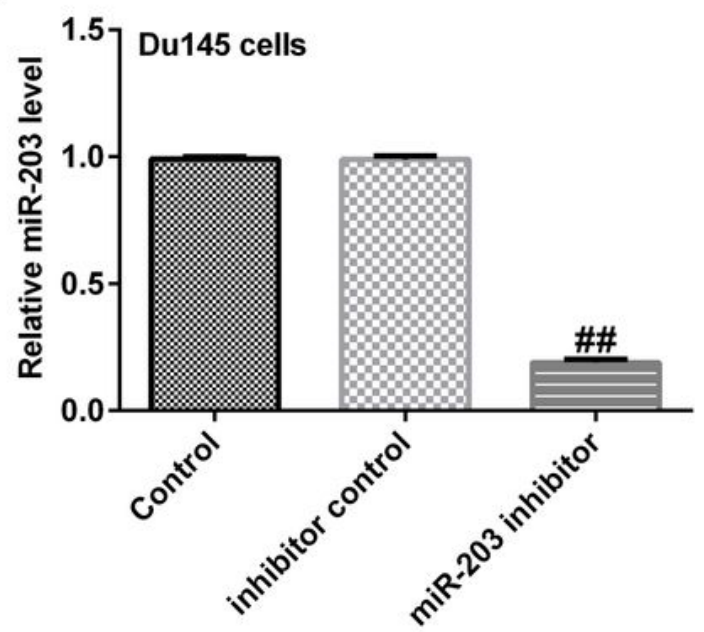

E

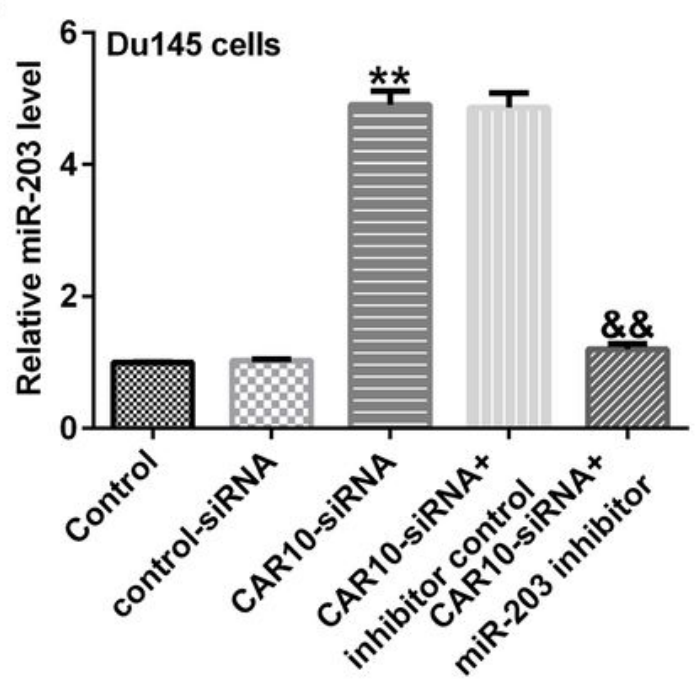

B

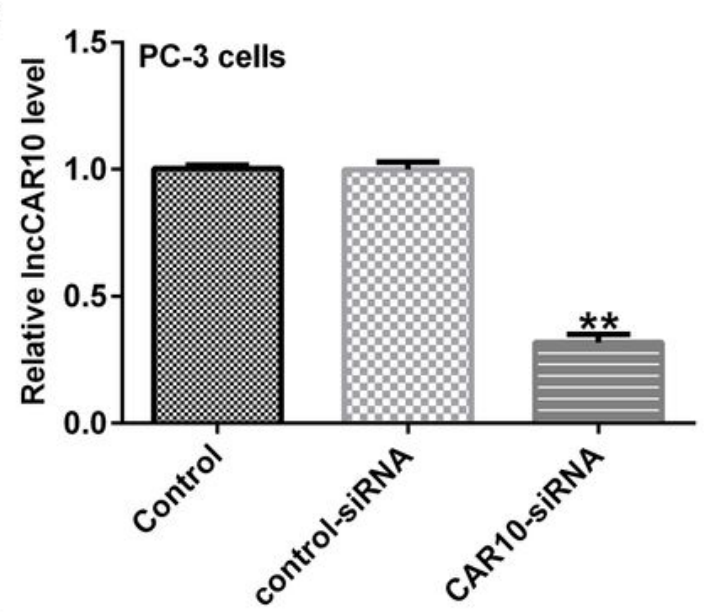

D

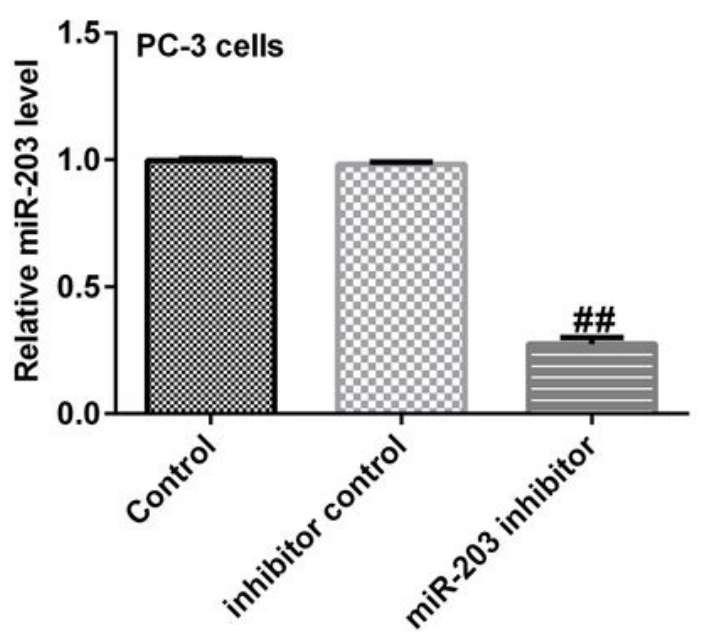

F

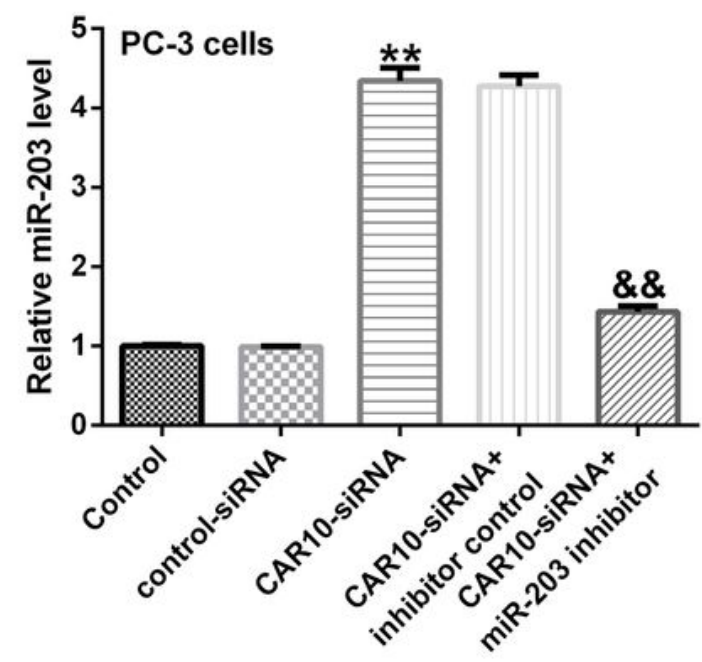

Figure 4

CAR10 negatively regulated miR-203 expression in PC cells. A and B: the effect of CAR10-siRNA on CAR10 expression in Du145 and PC-3 cells. C and D: the effect of miR-203 inhibitor on miR-203 expression in Du145 and PC-3 cells. E and F: the effect of CAR10-siRNA on miR-203 expression in Du145 and PC-3 cells. ${ }^{\star *} p<0.01$ vs. control-siRNA group; \#\#p<0.01 vs. inhibitor control group; \&\&p<0.01 vs. CAR10-siRNA+inhibitor control group. 


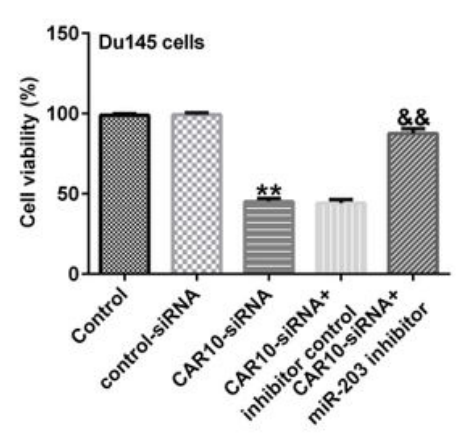

D

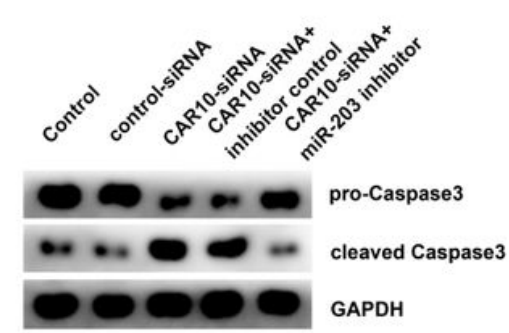

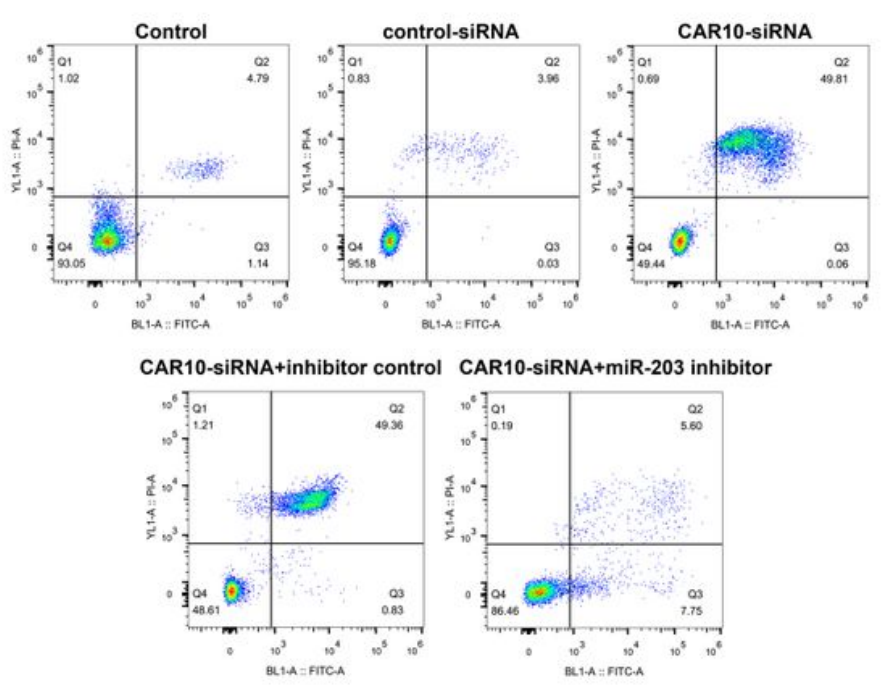

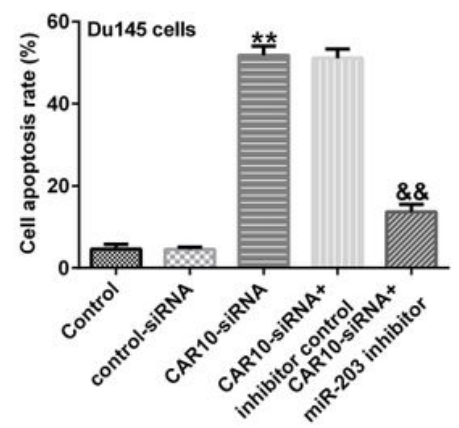

E

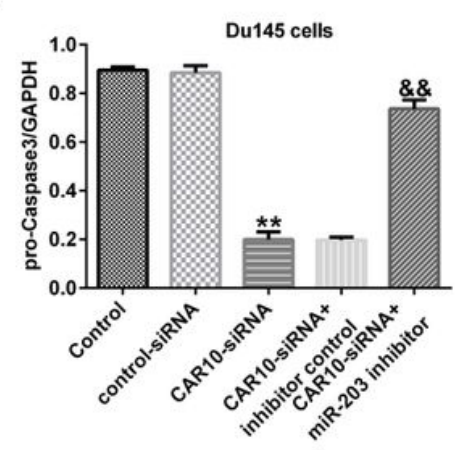

F

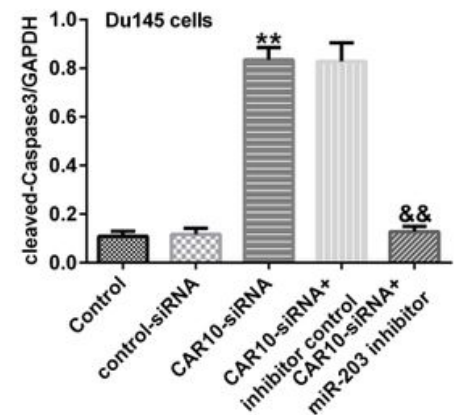

\section{Figure 5}

The effect of CAR10 down-regulation on Du145 cell viability and apoptosis. We used flow cytometry and MTT assay to detect Du145 cell viability and apoptosis, and used western blot assay to detect the expression of cleaved-caspase3 and pro-caspase3. A: the effect of CAR10-siRNA on the viability of Du145 cells. B and C: the effect of CAR10-siRNA on the apoptosis of Du145 cells. D-F: the effect of CAR10-siRNA on pro-caspase3 and cleaved-caspase3 protein expression in Du145 cells. Data were reported as mean $\pm S D$. ${ }^{\star \star} p<0.01$ vs. control-siRNA group; $\& \& p<0.01$ vs. CAR10-siRNA+inhibitor control group. 
A
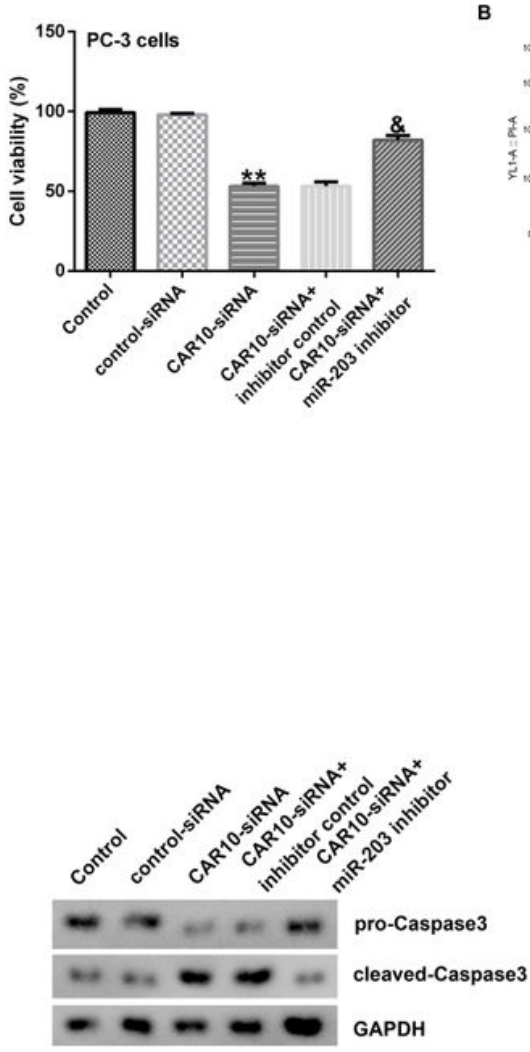

B

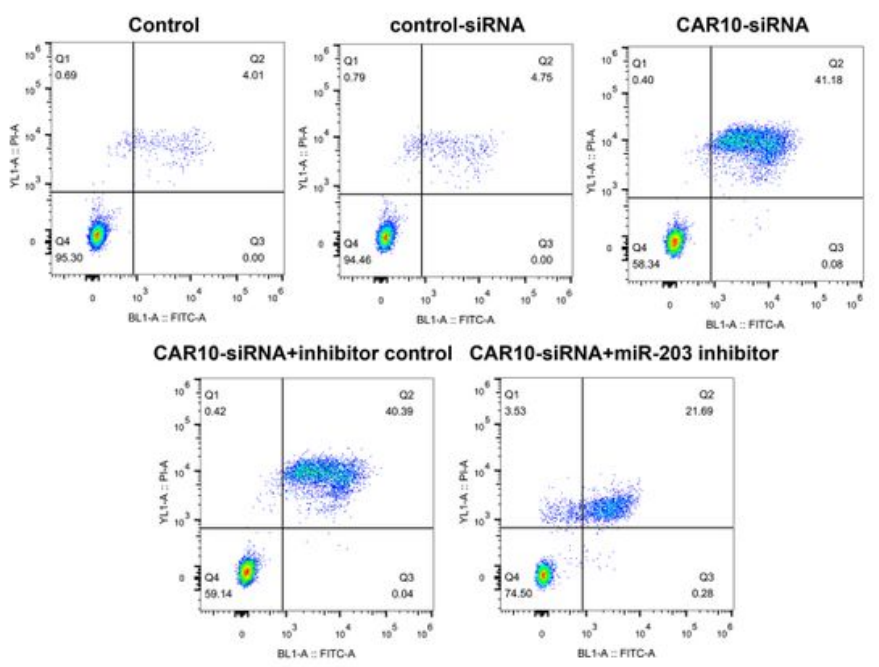

C

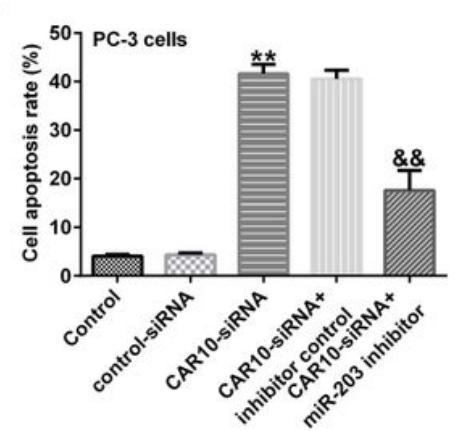

E

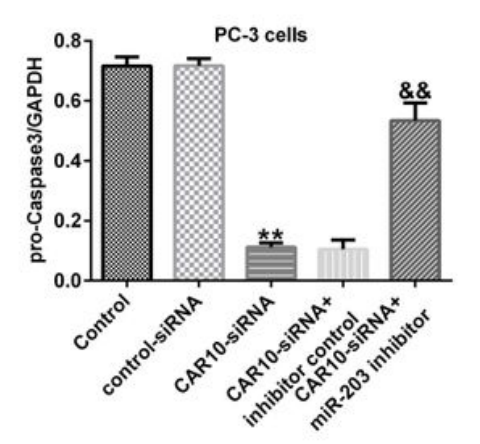

F

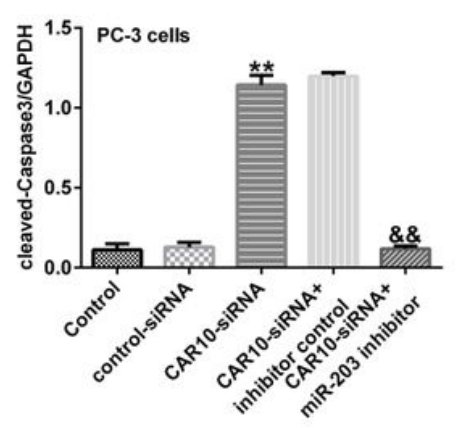

Figure 6

The effect of CAR10 down-regulation on PC-3 cell viability and apoptosis. We used flow cytometry and MTT assay to detect PC-3 cell viability and apoptosis, and used western blot assay to detect the expression of cleaved-caspase3 and pro-caspase3. A: the effect of CAR10-siRNA on the viability of PC-3 cells. B and C: the effect of CAR10-siRNA on the apoptosis of PC-3 cells. D-F: the effect of CAR10-siRNA on pro-caspase3 and cleaved-caspase3 protein expression in PC-3 cells. Data were reported as mean \pm SD. ${ }^{* *} p<0.01$ vs. control-siRNA group; $\&, \& \& p<0.05,0.01$ vs. CAR10-siRNA+inhibitor control group. 